\title{
Combined interstitial and surface high-dose-rate brachytherapy treatment of squamous cell carcinoma of the hand
}

\author{
Daniel W. Kim, MD, MBA!, Ivan M. Buzurovic, PhD², Brandon V. Mahal, MD!' William Hwang, MD, PhD!, \\ Oluwadamilola T. Oladeru, MD', Desmond A. O'Farrell, MSc², Thomas C. Harris, MS², Danielle N. Margalit, MD, MPH ${ }^{3}$, \\ Miranda Lam, MD, MBA², Phillip M. Devlin, MD² \\ 'Department of Radiation Oncology, Massachusetts General Hospital, Harvard Medical School, Boston, MA, United States, ${ }^{2}$ Department \\ of Radiation Oncology, Brigham and Women's Hospital, Dana-Farber Cancer Institute, Harvard Medical School, Boston, MA, United States, \\ ${ }^{3}$ Head and Neck Oncology Program, Dana-Farber Cancer Institute, Harvard Medical School, Boston, MA, United States
}

\begin{abstract}
Purpose: We present a case report of treatment using interstitial and surface high-dose-rate (HDR) brachytherapy for cutaneous squamous cell carcinoma (SCC) involving the interspace of the third and fourth digits. The patient refused two-ray amputation and the lesion was not amenable for external beam radiation therapy (EBRT). This is the first report detailing combined interstitial and surface HDR brachytherapy for a hand SCC.

Material and methods: The patient received 4050 cGy in 9 fractions, twice daily using 6 interstitial catheters and 8 Freiburg flap catheters. The clinical target was defined by MRI and ultrasound as a dorsal mass to the interspace between the heads of the third and fourth metacarpals measuring approximately $7 \mathrm{~mm}$ transverse $\times 5 \mathrm{~mm}$ volar-dorsal $\times 16 \mathrm{~mm}$ proximal-distal.

Results: The treatment resulted in radiographic and clinical tumor control. The patient retained functional use of her hand. However, there were both acute and late treatment-related side effects. Acutely, inpatient admission for pain control with a nerve block was needed. Long-term toxicity was notable for grade 2 skin necrosis treated with hyperbaric oxygen.

Conclusions: The first interstitial and surface HDR brachytherapy for cutaneous squamous cell carcinoma of a finger interspace for hand function preservation is presented. The initial experience revealed that brachytherapy was tolerated but with notable acute and late side effects. Treatment did result in tumor shrinkage with organ preservation and function of two rays. A larger cohort of patients will be required for additional conclusions related to long-term clinical benefits in patients who refuse ray amputation.

Key words: interstitial brachytherapy, surface brachytherapy, cutaneous squamous cell carcinoma.

\section{Purpose}

Squamous cell carcinoma (SCC) is a common malignancy derived from epidermal keratinocytes. It is estimated that over 700,000 new cases of SCC are diagnosed annually [1]. The most common presentation is localized, highly curable disease with locoregional or distant metastases developing in less than $5 \%$ of cases [2,3]. SCC can develop on any cutaneous surface although the most common sites are those exposed to the sun. The rate of SCC in the arm or hand is estimated at 3\% [4].

Treatment is primarily determined by risk of locoregional recurrence and metastasis. Treatment options include surgical excision, cryotherapy, electrosurgery, topical treatment, radiation therapy, and photodynamic therapy, with surgery being the most frequent treatment due to low rates of recurrence [5]. In terms of radiation therapy, different techniques can be used such as electron beams, megavoltage photons, low-dose-rate (LDR) or high-dose-rate (HDR) brachytherapy [6]. The treatment optionality is based on institutional resources and radiation oncologist expertise.

Radiation therapy is an effective alternative to surgery. While EBRT can be performed, tissues of the hand do not often tolerate cancercidal doses of radiation and damage to the skin and underlying tendons and bones may produce a poor cosmetic and functional result [7]. There is very limited literature regarding treatment of
Address for correspondence: Ivan M. Buzurovic, PhD, Department of Radiation Oncology, Brigham and Women's Hospital, Dana-Farber Cancer Institute, Harvard Medical School, 75 Francis St., L2, CWL214, 02115, Boston, MA, United States, phone: +1 617-278-0436, fax: +1 617-264-6358, e-mail: ibuzurovic@bwh.harvard.edu
Received: 23.08.2019

Accepted: 30.11 .2019

Published: 28.02.2020 
non-melanoma skin cancer using HDR brachytherapy. Also, these reports on HDR brachytherapy concern delivery via a surface mold and not interstitial catheters. Surface mold techniques have shown comparable tumor control to surgery with good cosmetic and functional outcomes $[8,9,10,11,12,13,14]$.

The role of interstitial brachytherapy for SCC involving the hand has not been well defined. The purpose of the study is to report the treatment of SCC using interstitial and surface HDR brachytherapy to obtain tumor control and preserve organ function. To our knowledge, this is the first such report in the literature that shows the use of interstitial catheters to deliver HDR brachytherapy for recurrent cutaneous SCC.

\section{Material and methods}

\section{Patient}

The patient was a 48-year-old woman who worked as a healthcare professional with a medical history notable for four renal transplantations for idiopathic renal failure beginning at age eight. She was on chronic immunosuppression with tacrolimus and mycophenolic acid. She presented initially with cutaneous SCC two years previously with a nodule of the dorsal right hand involving the $3^{\text {rd }}$ and $4^{\text {th }}$ webspace. Biopsy confirmed squamous cell carcinoma and she underwent Mohs resection of the mass with subsequent reconstruction with chest wall flap. Resection included at least partial excision of the ring finger radial digital nerve due to perineural invasion. A year later, she noted a recurrent mass of the same webspace and underwent re-resection with negative margins. Over the next several months, the mass slowly re-grew in the same webspace, prompting a biopsy with pathology consistent with recurrent SCC. Magnetic resonance imaging (MRI) at that time showed a dorsal mass to the interspace between the heads of the third and fourth metacarpals measuring approximately $7 \mathrm{~mm}$ transverse $\times 5 \mathrm{~mm}$ volar-dorsal $\times$ $16 \mathrm{~mm}$ proximal-distal. The tumor significantly involved the webspace with some penetration of the lesion into the volar neurovascular bundle. Clinically, there was suspi-

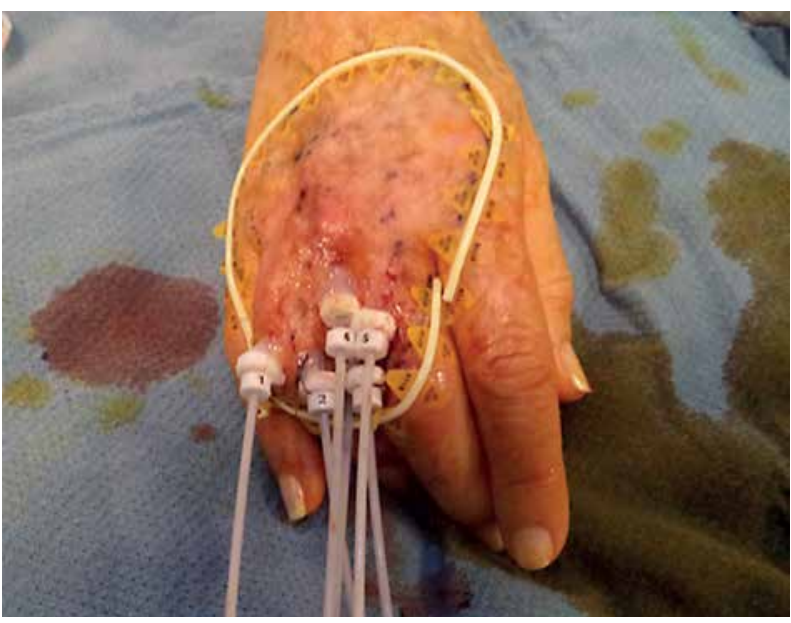

Fig. 1. Catheter insertion; radio-opaque marking wire is used to delineate superficial component of the CTV cion of dorsal, dermal invasion; however, MRI did not show bone invasion. She was seen in consultation at an outside institution where single $\left(4^{\text {th }}\right.$ digit $)$ to double ray resection $\left(3^{\text {rd }}\right.$ and $4^{\text {th }}$ digits) was recommended.

The patient presented to our institution for a second opinion. The recommendation of upfront surgical management was reinforced. As the patient refused ray amputation due to functional loss and cosmetic deformity, the option of initial external beam radiation therapy followed by surgical tumor debulking was discussed. The potential serious complications of surgery and radiation therapy were discussed in detail [15]. Due to the increased risk of wound healing complications, significant impairment of lymphatic drainage, fibrosis, swelling, and continued pain which could have limited the function of her hand, she declined this option as well. The option of EBRT alone using electrons was proposed. An initial plan using electrons was generated but the dose inhomogeneity was deemed unacceptable given the complexity of the target. Subsequently, she was referred to the brachytherapy group for consideration of interstitial brachytherapy (Figure 1). After much discussion, a plan of surface and interstitial HDR brachytherapy was generated with the goal of balancing tumor control with preserving organ function.

\section{Treatment planning}

The Oncentra Brachy (Ver. 4.3., Elekta Brachytherapy) treatment planning system (TPS) was used for 3D treatment plan generation. A CT image set with a $1.25 \mathrm{~mm}$ slice thickness was used to allow for accurate digitization of the catheter. The prescription dose for the HDR treatments was $40.5 \mathrm{~Gy}$ in 9 fractions to the clinical target volume (CTV) [7]. The CTV was defined by the wire for the surface part (as seen in Figure 1). The subcutaneous part of the target contains the interspace between the heads of the third and fourth metacarpals (as seen in Figure 2). The prescription dose was planned to be delivered using $5 \mathrm{~mm}$ step size sequencing. The applicator consists of the two components: a) flap applicator (Freiburg Flap, Elekta Brachytherapy) with 8 catheters, and b) six $1200 \mathrm{~mm}$ ProGuide (Elekta Brachytherapy) interstitial needles. The flap applicator was clinically placed at the surface of the

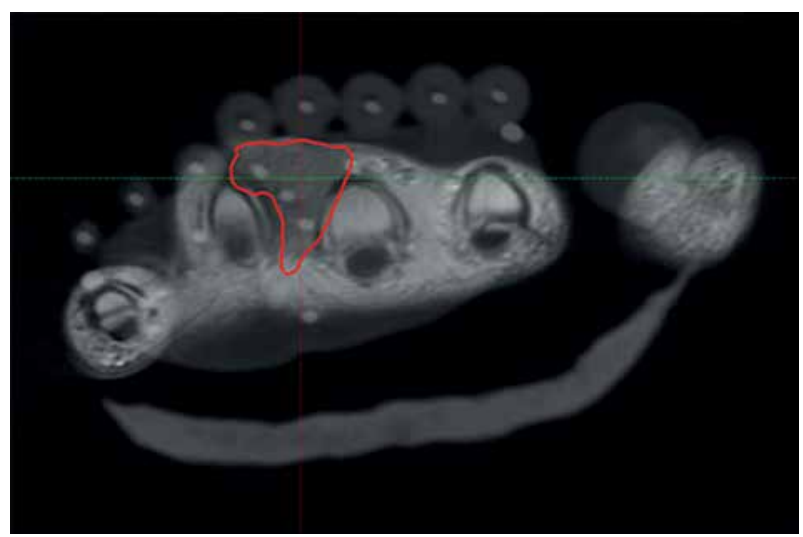

Fig. 2. MRI was used to evaluate interstitial tumor progression, and to define the CTV 
back of the hand. The flap applicator was used in a standard manner in the treatment planning phase to deliver the prescription dose to $3 \mathrm{~mm}$ under the skin to reach the dermis and spare unaffected stem cells and vasculature. Therefore, the treatment plan was initially normalized to the depth of $3 \mathrm{~mm}$ maintaining an equidistant position of $8 \mathrm{~mm}$ between the source dwell positions and normalization points. The interstitial needles were used to bring the dose to the depth forming the T-shape isodose distribution around the proximal phalanges (Figure 3). The distal position of the source in the TPS was determined using two independent measurements. The length of all 14 catheters was measured using the source position simulator (SPS). The length of marking wires for each catheter was compared against the values obtained with the SPS to reach a tolerance level of $1 \mathrm{~mm}$. Only dwell position inside the clinical target was activated to minimize the dose to the healthy tissue. Strictly local graphical optimization of the dose was applied due to the small size and complicated topology of the target. Paraxial, parasagittal, paracoronal views were used to evaluate dose distributions over the 3D volumes. The relevant dosimetric parameters such as $\mathrm{V}_{100}$, $\mathrm{V}_{150}, \mathrm{~V}_{200}, \mathrm{D}_{90}$ and $\mathrm{D}_{95}$ for the CTV were collected. In addition, $\mathrm{V}_{100}, \mathrm{~V}_{150}, \mathrm{~V}_{200}$, and $\mathrm{D}_{2 \mathrm{~cm} 3}$ for the bone were recorded to evaluate the patient dosimetry. The dose calculation was performed using the TG-43 formalism that includes the anisotropy corrections. Heterogeneity corrections were not included in the brachytherapy dose calculation.

\section{Results}

\section{Treatment delivery}

To minimize uncertainties during immobilization, self-adherent wraps, and towels for additional support were used (Figure 4). The setup was verified against the clinical photograph taken on the day of simulation. The position of the flap applicator with respect to the interstitial needles was verified prior to each fraction using CT images. The planning CT images were compared against the pretreatment CT images. Pretreatment CT images were used to evaluate possible gaps between the skin and surface applicator. The treatment plan was generated to maintain a maximum dose at the skin of $125 \%$ of the prescription dose, so the position of the applicator was carefully verified to maintain the desired dosimetry. The patients tolerated the treatment well in the sitting position. The patient did not experience discomfort during pretreatment preparation or during the delivery of the radiation dose. The treatment time for the last $\left(9^{\text {th }}\right)$ fraction was $403 \mathrm{~s}$, which was $20 \mathrm{~s}$ longer relative to the first received treatment. Therefore, the extended number of fractions did not result in a significant increase of the treatment time.

Total treatment time was $383 \mathrm{~s}$ when the source activity was 6.2 Ci (air kerma strength equals $25.208 \mathrm{mGy}-$ $\left.\mathrm{m}^{2}-\mathrm{h}^{-1}\right)$. The total time lost due to rounding of the dwell times was negligible $(0.7 \%)$. All 8 catheters from the flap applicator contributed to the total dose uniformly - the dwell times range from 34.1 to $48.2 \mathrm{~s}$. The dwell times for the interstitial needles were between 2.7 and $25.2 \mathrm{~s}$. Treatment times for superficial and interstitial component of the treatment plan were $84 \%$ and $16 \%$ of total treatment time, respectively. This was to be expected since the interstitial component of the applicator was used for fine distribution of the dose to the clinical target to allow for adequate coverage. Dosimetry of the CTV and bone is presented in Table 1. It was found that $D_{90}$ and $D_{95}$ for the CTV was $100.13 \%$ and $95.95 \%$, respectively. $V_{100}$ for the bone was $0.82 \%$ or $0.63 \mathrm{~cm}^{3}$. $\mathrm{V}_{150}$ and $\mathrm{V}_{200}$ for the bone were found to be 0 .

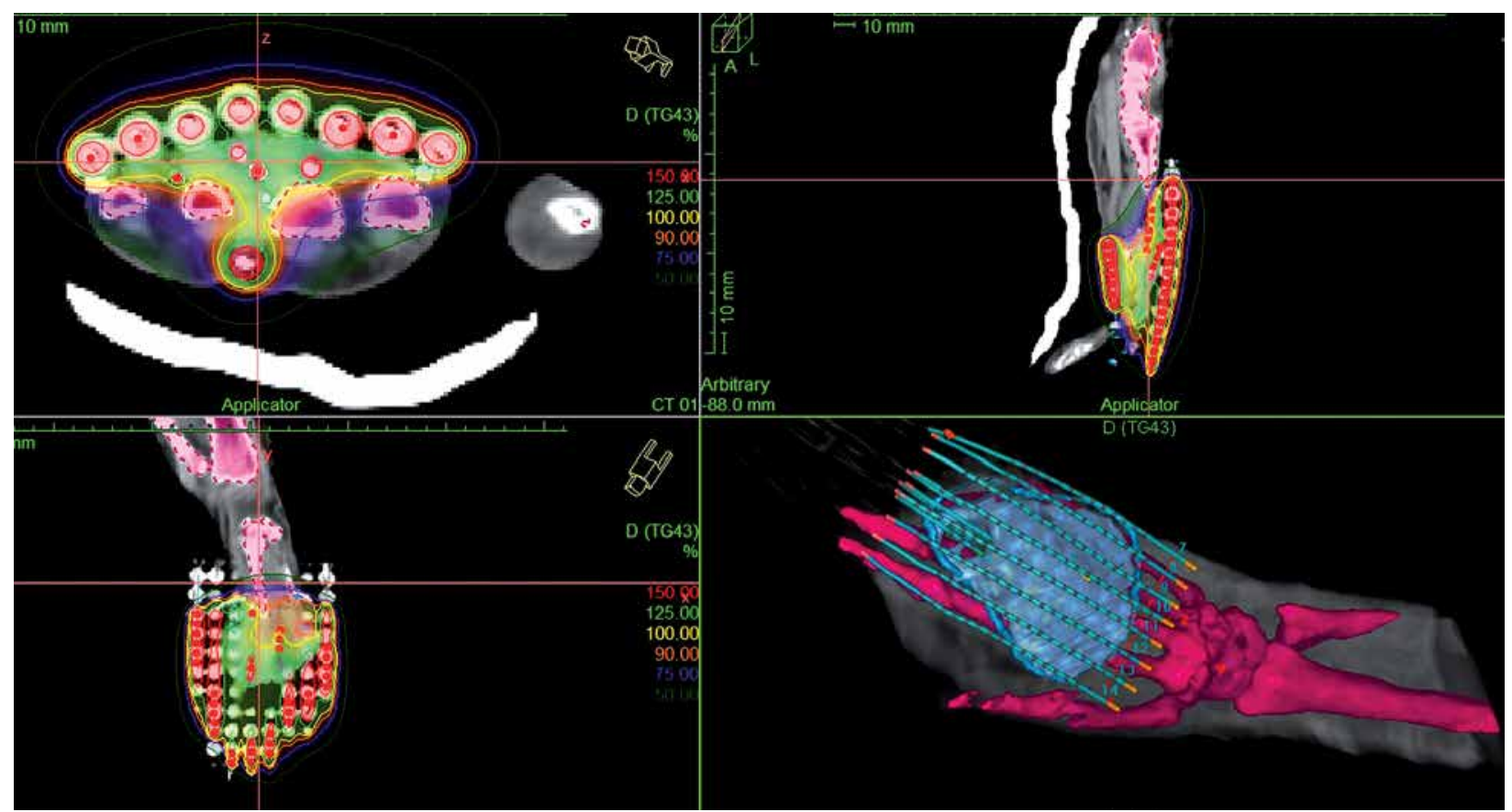

Fig. 3. Dosimetry and planning; axial slice shows T-shape dose distribution due to contribution to the dosimetry introduced by interstitial needles 
Overall, the patient tolerated the whole treatment course. She required hospitalization one day after insertion of the catheters for pain control with an axillary block. After treatment, she was started on tramadol and gabapentin for long-term pain management. At threemonth follow-up, MRI showed a small interval decrease in tumor size with resolution of her pain. In addition, the patient retained functional use of her $3^{\text {rd }}$ and $4^{\text {th }}$ digits. She was able to continue her work as a healthcare professional during this post-treatment period. Her six-month MRI showed an interval decrease in the tumor size as well as a newly developed grade 2 soft tissue necrosis that was treated with hyperbaric oxygen.

\section{Discussion}

The standard of care of SCC involving the hand and/ or fingers is largely surgery. This is based on literature showing high tumor control rates after surgical excision $[16,17,18]$. Two of these series cite a recurrence rate as low as 3\% [16,17]. Schiavon et al. reported 5-year progression-free survival of $82 \%$ [18]. The main reasons for radiation therapy for SCC have been inoperability either due to the patient's inability to tolerate surgery and/or reconstruction, size and location or lesion, and patient preference. Finally, although long-term cosmesis after surgery, especially with the rise of Mohs micrographic surgery, has improved, radiotherapy often offers superb cosmetic results $[8,9,10,11,12,13,14]$.

The 5-year-control rates for non-melanoma skins cancers of all sites treated with EBRT are good, ranging from $80 \%$ to $93 \%$ [19]. Brachytherapy provides the distinct advantage of maximizing the dose to the tumor while minimizing delivery of the dose to normal tissue. To individualize treatment parameters for a patient's tumor, surface molds and/or interstitial catheters are used to position the isotope and deliver precisely targeted radiation.

The majority of the limited literature on brachytherapy for nonmelanoma skin cancer involves HDR surface mold modality [20]. There are only four reports that report interstitial LDR brachytherapy and all involve sites of the face $[21,22,23,24]$. Radiobiological studies showed that the effectiveness of LDR and HDR protocols was the

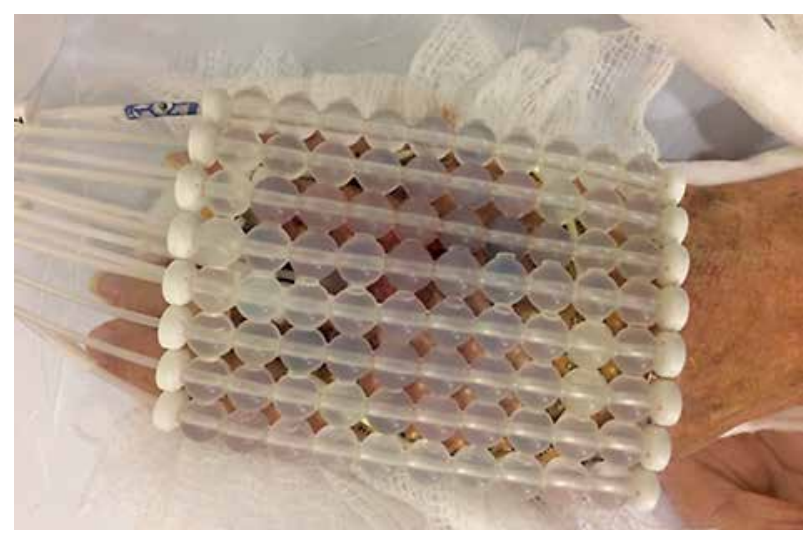

Fig. 4. Treatment delivery setup (prior to immobilization using self-adherent wrap). Freiburg flap applicator is placed to the surface of the CTV

same without increasing toxicity [25]. There is no literature that we are aware of that reports on interstitial HDR brachytherapy for SCC of the hand.

The primary goal of this treatment was to prevent the growth of the tumor and preserve the function and cosmesis of the patient's hand. With interstitial and surface HDR brachytherapy, we were able to achieve both objectives albeit with notable side effects. Tumor control assessed by MRI at three and six months showed $~ 10 \%$ decrease in tumor size. Treatment-related side effects acutely were pain and long-term skin necrosis. While the patient did require hospitalization for an axillary block for pain control, this was temporary and her pain returned to baseline within two weeks after treatment. Given the location of the tumor and concern for dorsal, dermal invasion, the dose of the superficial skin was weighed against the potential for skin necrosis. In this patient, grade 2 skin necrosis was observed and treated with hyperbaric oxygen. From this patient's perspective, the cosmetic result was more than acceptable when weighed against amputation. When planning these cases, the balance between achieving a tumoricidal dose and potential side effects will be an ongoing challenge. Patients should be counseled on potential side effects and the potential for long-term skin necrosis. No complications or tumor

Table 1. Dosimetry of the CTV and finger bones

\begin{tabular}{|c|c|c|c|c|}
\hline ROI & Dose (\%) & Dose (cGy) & Volume (\%) & Volume $\left(\mathrm{cm}^{3}\right)$ \\
\hline CTV & 100 & 450 & 90.2 & 11.32 \\
\hline CTV & 150 & 675 & 6.17 & 0.77 \\
\hline CTV & 200 & 900 & 2.56 & 0.32 \\
\hline CTV & 100.13 & 450.58 & 90 & 11.29 \\
\hline CTV & 95.95 & 431.77 & 95 & 11.92 \\
\hline Bone & 100 & 450 & 0.82 & 0.63 \\
\hline Bone & 150 & 675 & 0 & 0 \\
\hline Bone & 200 & 900 & 0 & 0 \\
\hline Bone & 94.09 & 423.39 & 2.58 & 2 \\
\hline
\end{tabular}




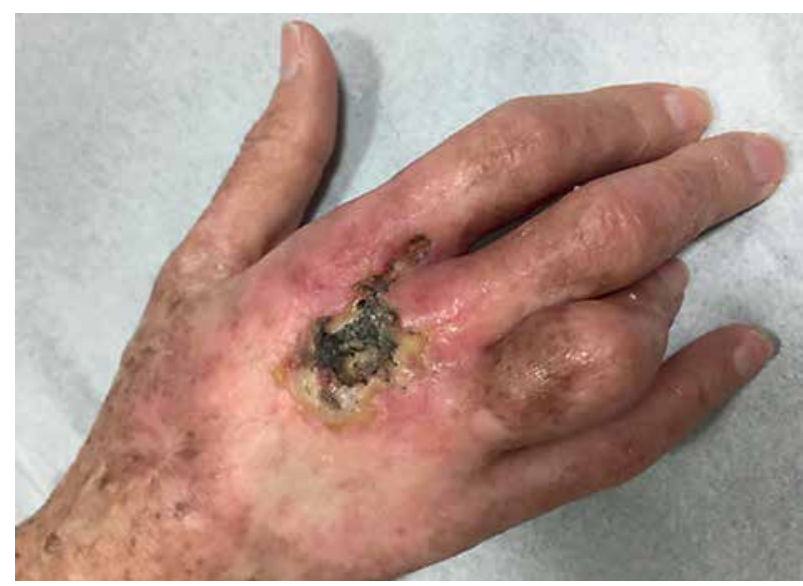

Fig. 5. Six-month post-treatment follow-up; no tumor progression was noticed

progression were noticed at our standard follow-ups at 2 weeks, 8 weeks, six months (Figure 5) and one year. The patient remains under regular surveillance. In summary, the treatment allowed the patient to retain functional use of her hand and produced an acceptable cosmetic outcome while providing tumor control. In selected patients who refuse ray amputation, this treatment paradigm could offer an alternative therapeutic option.

In the future, a prospective study should be conducted to evaluate the longer-term efficacy of tumor control and side effects in a larger set of patients. Also, as more patients are treated with this novel technique, case-matched studies with EBRT and/or surgery should be done.

\section{Conclusions}

In this case report we show that combined interstitial and surface HDR brachytherapy is an alternative therapy to ray amputation for a patient with recurrent SCC of the hand. Skin toxicity using this modality was observed but hand function after treatment is excellent. In carefully selected patients, interstitial and surface HDR brachytherapy can be an appropriate and effective option for recurrent SCC of the hand.

\section{Disclosure}

The authors report no conflict of interest.

\section{References}

1. Karia PS, Han J, Schmults CD. Cutaneous squamous cell carcinoma: estimated incidence of disease, nodal metastasis, and deaths from disease in the United States, 2012. J Am Acad Dermatol 2013; 68: 957-966.

2. Brantsch KD, Meisner C, Schönfisch B et al. Analysis of risk factors determining prognosis of cutaneous squamous-cell carcinoma: a prospective study. Lancet Oncol 2008; 9: 713-720.

3. Samarasinghe V, Madan V, Lear JT. Management of highrisk squamous cell carcinoma of the skin. Expert Rev Anticancer Ther 2011; 11: 763-769.

4. English DR, Armstrong BK, Kricker A et al. Demographic characteristics, pigmentary and cutaneous risk factors for squamous cell carcinoma of the skin: a case-control study. Int J Cancer 1998; 76: 628-634.
5. Chren MM, Torres JS, Stuart SE et al. Recurrence after treatment of nonmelanoma skin cancer: a prospective cohort study. Arch Dermatol 2011; 147: 540-546.

6. Delishaj D, Rembielak A, Manfredi B et al. Non-melanoma skin cancer treated with high-dose-rate brachytherapy: a review of literature. J Contemp Brachytherapy 2016; 8: 533-540.

7. Somanchi BV, Stanton A, Webb M et al. Hand function after high dose rate brachytherapy for squamous cell carcinoma of the skin of the hand. Clin Oncol (R Coll Radiol) 2008; 20: 691-697.

8. Gauden R, Pracy M, Avery AM et al. HDR brachytherapy for superficial non-melanoma skin cancers. J Med Imaging Radiat Oncol 2013; 57: 212-217.

9. Svoboda VH, Kovarik J, Morris F. High dose-rate microselectron molds in the treatment of skin tumors. Int J Radiat Oncol Biol Phys 1995; 31: 967-972.

10. Köhler-Brock A, Prager W, Pohlmann S et al. The indications for and results of HDR afterloading therapy in diseases of the skin and mucosa with standardized surface applicators (the Leipzig applicator). Strahlenther Onkol 1999; 175: 170-174.

11. Guix B, Finestres F, Tello J et al. Treatment of skin carcinomas of the face by high-dose-rate brachytherapy and custom-made surface molds. Int J Radiat Oncol Biol Phys 2000; 47: 95-102.

12. Guinot JL, Rembielak A, Perez-Calatayud J et al. GECESTRO ACROP recommendations in skin brachytherapy. Radiother Oncol 2018; 126: 377-385.

13. Ouhib Z, Kasper M, Perez Calatayud J et al. Aspects of dosimetry and clinical practice of skin brachytherapy: The American Brachytherapy Society working group report. Brachytherapy 2015; 14: 840-858.

14. Murakami N, Ueno $T$, Yatsuoka $W$ et al. Dose coverage comparison between "interstitial catheter-only" and "hybrid intracavitary-interstitial brachytherapy" for early stage squamous cell carcinoma of the buccal mucosa. J Contemp Brachytherapy 2018; 10: 486-491.

15. Spierer MM, Alektiar KM, Zelefsky MJ et al. Tolerance of tissue transfers to adjuvant radiation therapy in primary soft tissue sarcoma of the extremity. Int J Radiat Oncol Biol Phys 2003; 56: 1112-1116.

16. Forsythe RL, Bajaj P, Engeron O et al. The treatment of squamous cell carcinoma of the hand. Hand 1978; 10: 104-108.

17. Bean D, Reese R, O'Leary J et al. Carcinoma of the hand: a 20-year experience. South Med J 1984; 77: 998-1000.

18. Schiavon M, Mazzoleni F, Chiarelli A et al. Squamous cell carcinoma of the hand: Fifty-five case reports. J Hand Surg 1988; 13: 401-404.

19. Locke J, Karimpour S, Young G et al. Radiotherapy for epithelial skin cancer. Int J Radiat Oncol 2001; 51: 748-755.

20. Alam M, Nanda S, Mittal BB et al. The use of brachytherapy in the treatment of nonmelanoma skin cancer: a review. J Am Acad Dermatol 2011; 65: 377-388.

21. Conill C, Verger E, Marruecos J et al. Low dose rate brachytherapy in lip carcinoma. Clin Transl Oncol 2007; 9: 251-254.

22. Conill C, Sánchez-Reyes A, Molla M et al. Brachytherapy with 192Ir as treatment of carcinoma of the tarsal structure of the eyelid. Int J Radiat Oncol 2004; 59: 1326-1329.

23. Rio E, Bardet E, Ferron $C$ et al. Interstitial brachytherapy of periorificial skin carcinomas of the face: A retrospective study of 97 cases. Int J Radiat Oncol 2005; 63: 753-757.

24. Avril MF, Auperin A, Margulis A et al. Basal cell carcinoma of the face: surgery or radiotherapy? Results of a randomized study. Br J Cancer 1997; 76: 100-106.

25. Manimaran S. Radiobiological equivalent of low/high dose rate brachytherapy and evaluation of tumor and normal responses to the dose. Radiat Med 2007; 25: 229-235. 